\title{
Pharmacological Characterization of the Prostanoid Receptor EP3 in Locus Coeruleus Neurons by Single-Unit Extracellular Recordings in the Rat Brain in Vitro
}

\author{
Amaia Nazabal ${ }^{1}$, Aitziber Mendiguren ${ }^{1}$ and Joseba Pineda ${ }^{1 *}$
}

1 Department of Pharmacology, Faculty of Medicine and Odontology, University of the Basque Country (UPV/EHU), E-48940 Leioa, Bizkaia, Spain

* Author to whom correspondence should be addressed; e-mail: joseba.pineda@ehu.es

Published: 4 December 2015

\begin{abstract}
Prostanoids are known to regulate several physiological functions and to play an important role in certain pathophysiological situations such as inflammation. Prostaglandin $\mathrm{E}_{2}$ receptors (EP) are members of the $\mathrm{G}$ protein-coupled receptor superfamily. Four subtypes have been described: EP2 and EP4 (coupled to $\mathrm{G}_{\mathrm{s}}$ proteins) and EP1 and EP3 (coupled to Gi/o proteins). To date, the function of the prostanoid system in the brain has not been well characterized. The locus coeruleus (LC), the main noradrenergic nucleus in the brain, has been described to express the EP3 receptor. The aim of this study was to characterize the functional relevance of EP3 receptors in the LC by single-unit extracellular recordings in rat brain slices. We performed concentration-effect curves for different endogenous derivatives and selective agonists of EP3 receptors. Thus, increasing concentrations of the EP3/EP1 agonist sulprostone (0.3-80 nM) fully inhibited the neuronal activity of LC cells, with an EC 50 value of $15 \mathrm{nM}(\mathrm{n}=$ 9). The EP3 receptor antagonist L-798,106 $(10 \mu \mathrm{M})$ caused a rightward shift ( $>8$ fold) in the concentration-effect curve for sulprostone, but the EP2 receptor antagonist PF04418948 (10 $\mu \mathrm{M})$ or the EP4 receptor antagonist L-161,982 $(10 \mu \mathrm{M})$ failed to cause any rightward shift of sulprostone effect. On the other hand, perfusion with the endogenous $\mathrm{PGE}_{2}(0.3 \mathrm{nM}-1.28 \mu \mathrm{M})$ or the PGE1 analogue misoprostol $(0.3-320 \mathrm{nM})$ induced a concentration-dependent inhibition of the firing rate of LC cells, with EC50 values being $51 \mathrm{nM}$ and $112 \mathrm{nM}$, respectively. Likewise, only the EP3 antagonist L-798,106 $(10 \mu \mathrm{M})$ caused a rightward shift ( $>8$ fold) in the concentration-effect curves for these prostanoid agonists $(n=5)$. In conclusion, LC neurons are regulated in an inhibitory manner by the prostanoid system likely through the EP3 receptor.
\end{abstract}

Keywords: in vitro; locus coeruleus; electrophysiology; EP3 receptor; prostanoid; PGE2; sulprostone; slice 


\section{Introduction}

Prostanoids system is involved in the regulation of pain, fever and inflammation processes. During inflammation, membrane phospholipids are transformed into arachidonic acid (AA) by the phospholipase $A_{2}$ enzyme. In turn, AA is metabolized by cyclooxygenase $(\mathrm{COX})$ into $\mathrm{PGH}_{2}$, which is the common prostanoid precursor of other prostaglandins including prostacyclins and thromboxanes (Yagami et al. 2015). COX-blockers or NSAIDs -nonsteroidal anti-inflammatory drug- have been widely used as analgesic, antipyretic and antiinflammatory drugs due to their ability to suppress the production of prostanoids.

Two isoforms of COX (-1 and -2) have been characterized, but the isoform that has been shown to be constitutively expressed in the body is the isoform 1. However, recent studies have suggested that COX-2 could also be constitutively expressed in human and animal brain (Hétu \& Riendeau 2005; Martin et al. 2007; Yaksh et al. 2001). This supports the idea of a possible important role of prostaglandins in the central nervous system. Nevertheless, the function of prostaglandins remains unclear since dual effects have been described in the brain. On one hand, $\mathrm{PGE}_{2}$ seems to promote neuroprotection in injured brain by induction of either BDNF release or reducing the expression of inducible oxide nitric synthase (iNOS) during inflammation (Hutchinson et al. 2009; Levi et al. 1998). On the other hand, EP3 antagonists exert protective effects in the brain after ischemia (Ikeda-Matsuo et al. 2011).

$\mathrm{PGE}_{2}$ is the most abundant prostaglandin produced in the body and it acts through activation of EP1-4 receptors, which are expressed in neurons and glia (Ito et al. 2001). Classically, EP1 has been described to be coupled to $\mathrm{G}_{\mathrm{i} / \mathrm{o}}$ protein ( $\mathrm{Ji}$ et al. 2010), although there is recent evidence that supports its coupling to $\mathrm{G}_{\mathrm{q}}$ protein (Liu et al. 2010). EP3 is coupled to $\mathrm{G}_{\mathrm{i} / \mathrm{o}}$ protein (Negishi et al. 1995), whereas EP2 and EP4 are coupled to $\mathrm{G}_{\mathrm{s}}$ protein (Fujino \& Regan 2006; Sugimoto \& Narumiya 2007).

The locus coeruleus (LC) is the main source of noradrenergic innervation in the brain. It is involved in the regulation of numerous physiological functions such as sleep-wake cycle, arousal, cognition/memory, pain, cardiovascular control and rewarding behavior. A role of this nucleus in the production of fever has also been proposed (Almeida et al. 2004).

Several findings have suggested an interaction between the noradrenergic/LC system and prostanoid system. First, in situ hybridization experiments have demonstrated the presence of mRNA for the EP2 and EP4 receptors in this nucleus (Zhang \& Rivest 1999). Furthermore, EP3 expression has also been shown by inmunoreactivity and double hybridization techniques (Ek et al. 2000; Nakagawa et al. 2000; Nakamura et al. 2001). Second, the LC has been identified as a pivotal nucleus in PGE2induced thermogenesis (Almeida et al. 2004). Third, administration of PGE2 inhibits, via EP3 receptors the release of noradrenaline in the brain (Exner \& Schlicker 1995).

Despite several evidences that suggest a possible role of the prostanoid system in the LC, the functional role of EP receptors in this nucleus remained to be studied. Therefore, the aim of our research was to characterize, by single-unit extracellular recordings in vitro, the functional role of the prostanoid receptor EP3 in LC neurons from the rat brain. For this purpose, we performed concentration-effect curves for several EP3 receptor agonists in the absence and in the presence of different EP receptor antagonists. 


\section{Results and Discussion}

Effect of the EP3/EP1 receptor agonist sulprostone on the firing rate of $L C$ neurons

To evaluate the effect of prostaglandins on the LC neurons, we applied increasing concentrations of the EP3/EP1 agonist sulprostone $(0.3-80 \mathrm{nM})$ and we found that it fully inhibited the neuronal activity of LC cells, with an $\mathrm{EC}_{50}$ value of $15 \mathrm{nM}(\mathrm{n}=9)$. To identify the EP receptor involved in the inhibitory effect induced by sulprostone, we performed the concentration-effect curves for the EP3/EP1 agonist in the presence of specific antagonists of the EP receptors expressed in the LC: EP2 (PF04418948), EP3 (L-798,106) and EP4 (L161,982) at 3 and $10 \mu \mathrm{M}$. The EP3 receptor antagonist L-798,106 $(10 \mu \mathrm{M})$ caused a rightward shift ( $>8$ fold) in the concentration effect curve for sulprostone (Fig. 1). Neither the EP2 receptor antagonist PF04418948 $(10 \mu \mathrm{M})$ nor the EP4 receptor antagonist L-161,982 (10 $\mu \mathrm{M})$ caused any rightward shift of sulprostone effect.

These results indicate that the inhibitory effect of sulprostone is mediated by the EP3 receptor, which is known to be coupled to $\mathrm{G}_{\mathrm{i} / \mathrm{o}}$ proteins. The inhibitory effect mediated by the EP3 receptor has been shown to occur in other nucleus at the same concentrations used in our study (Ito et al. 2000). Therefore, our results show that EP3 receptor is functionally active in the LC. Future experiments are required to characterize the relevance of other EP receptors to the modulation of the firing activity of LC cells.

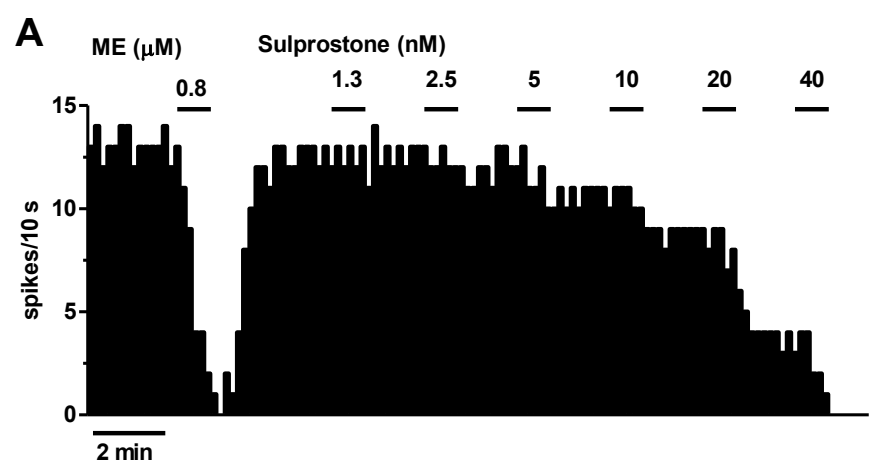

B

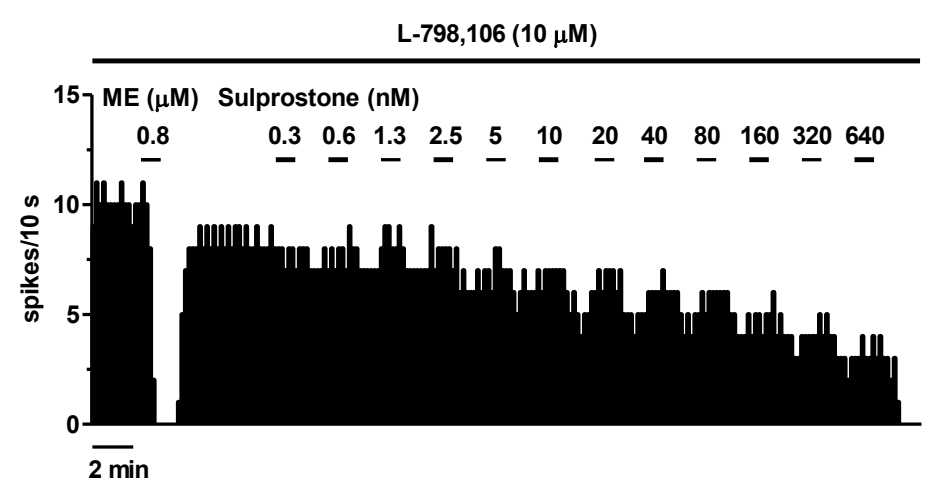

Figure 1. Effect of the EP3/EP1 receptor agonist sulprostone on the firing rate of locus coeruleus cells in the absence and in the presence of the EP3 receptor antagonist L-798,106. Representative examples of firing-rate recordings of two LC cells showing the effect of increasing concentrations of sulprostone in the 
$\mathrm{http}: / /$ sciforum.net/conference/mol2net-1

absence (A) and in the presence (B) of the EP3 antagonist L-798,106. Each horizontal bar represents the period of application of each sulprostone concentration and the vertical lines show the number of spikes recorded every $10 \mathrm{~s}$. The inhibitory effect induced by each application was calculated as a percentage from the basal firing rate. Note that sulprostone inhibits the firing rate of LC cells and that its inhibitory effect is diminished in the presence of L-798,106.

Effect of the endogenous $P G E_{2}$ and the $P G E_{1}$ analogue misoprostol on the firing rate of $L C$ neurons

In order to study whether endogenous prostanoid compounds mimicked the effect observed with sulprostone on the LC, we applied the endogenous $\mathrm{PGE}_{2}$ and the $\mathrm{PGE}_{1}$ analogue misoprostol. Perfusion with the endogenous $\mathrm{PGE}_{2}(0.3 \mathrm{nM}-1.28 \mu \mathrm{M})$ or the $\mathrm{PGE}_{1}$ analogue misoprostol (0.3-320 nM) induced a concentration-dependent inhibition of the firing rate of LC cells, with $\mathrm{EC}_{50}$ values being $51 \mathrm{nM}$ and $112 \mathrm{nM}$ respectively. Likewise, only the EP3 antagonist L-798,106 $(10 \mu \mathrm{M})$ caused a rightward shift ( $>8$ fold) in the concentration-effect curves for these prostanoid agonists (Fig. 2; $\mathrm{n}=5$ ).

These results indicate that sulprostone and the endogenous derivatives act through activation of the same EP receptor; the EP3 receptor. However, sulprostone shows higher potency than the endogenous prostanoid derivatives to inhibit LC cells.

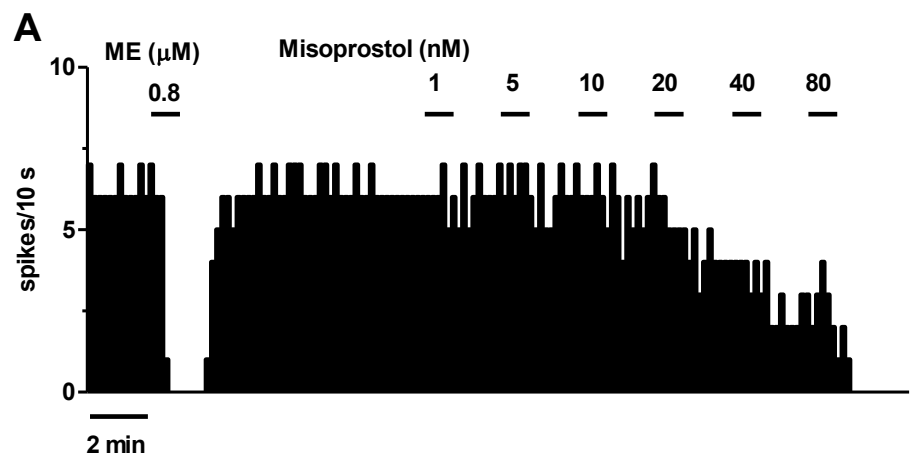

B

L-798,106 (10 $\mu \mathrm{M})$

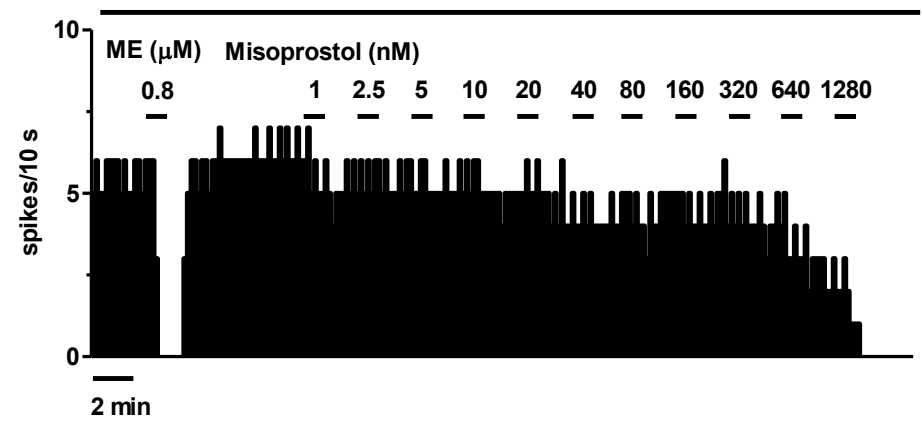

Figure 2. Effect of the $\mathrm{PGE}_{1}$ analogue misoprostol on the firing rate of locus coeruleus cells in the absence and in the presence the EP3 receptor antagonist L-798,106. Representative examples of firing-rate recordings of two LC cells showing the effect of increasing concentrations of misoprostol in the absence (A) and in the presence (B) of the EP3 antagonist L-798,106. Each horizontal bar represents the period of application of each misoprostol concentration and the vertical lines show the number of spikes recorded every $10 \mathrm{~s}$. The inhibitory effect induced by each application was calculated as a percentage from the basal firing rate. Note that misoprostol inhibits the firing rate of LC cells and that its inhibitory effect is diminished in the presence of L-798,106. 


\section{Materials and Methods}

\subsection{Animals}

Adult male Sprague-Dawley rats weighing 200$300 \mathrm{~g}$ were housed under controlled laboratory conditions $\left(22^{\circ} \mathrm{C}\right.$ and 12-h light/dark cycles) with free access to food and water. The animals were obtained from the animal house of the University of the Basque Country (Leioa, Spain). All experimental procedures reported in this manuscript were conducted in accordance with U.K. Animals (Scientific Procedures) Act, 1986, and associated guidelines, and with the European Community Council Directive on "Protection of Animals Used in Experimental and Other Scientific Purposes" of 24 November 1986 (86/609/EEC). The procedures were approved by the Animal Care and Use Committee of the University of the Basque Country. All efforts were made to minimize animal suffering and to reduce the number of animals used.

\subsection{In vitro electrophysiology}

\subsubsection{Brain slice preparation}

In vitro experiments were performed as previously described (Mendiguren \& Pineda 2007). Briefly, animals were first anaesthetized with chloral hydrate (400 mg/kg, i.p.) and sacrificed by decapitation. The brain was removed and a block of tissue containing the brainstem was placed in ice-cold modified artificial cerebrospinal fluid (aCSF) where $\mathrm{NaCl}$ was equiosmotically substituted with sucrose to improve neuronal viability. Coronal slices of 600 $\mu \mathrm{m}$ thickness containing the $\mathrm{LC}$ were cut by an oscillating vibratome and then allowed to recover from the slicing for $90 \mathrm{~min}$ in oxygenated aCSF. Next, slices were placed on a nylon mesh and maintained at $33 \pm 0.5 \mathrm{C}$ in a modified Haas-type interface chamber continuously perfused with oxygenated aCSF $\left(95 \% \mathrm{O}_{2} / 5 \% \mathrm{CO}_{2}, \mathrm{pH}=7.38\right)$ at a flow rate of $1-1.5 \mathrm{ml} / \mathrm{min}$. The aCSF contained (in $\mathrm{mM}$ ): $\mathrm{NaCl} 130, \mathrm{KCl} 3, \mathrm{NaH}_{2} \mathrm{PO}_{4}$ 1.25, Dglucose 10, $\mathrm{NaHCO}_{3} 21, \mathrm{CaCl}_{2} 2$, and $\mathrm{MgSO}_{4} 2$.

\subsubsection{Recording procedures}

Extracellular recordings of single neurons were performed as previously described (Mendiguren $\&$ Pineda 2004). The recording electrode consisted of an Omegadot glass micropipette that was pulled and filled with a solution of $50 \mathrm{mM}$ $\mathrm{NaCl}$ (tip size of 2-5 $\mu \mathrm{m}, 3-5 \mathrm{M} \Omega$ ). The microelectrode was placed in the $\mathrm{LC}$, which was visually identified in the rostral pons as a dark oval area on the lateral borders of the central gray and the 4th ventricle, just anterior to the genu of the facial nerve. The extracellular signal recorded by the microelectrode was passed through a high-input impedance amplifier system and monitored on an oscilloscope and by an audioanalyzer. Individual (single-unit) neuronal spikes were isolated from the background noise with a window discriminator and counted. The firing rate was represented and analyzed by a PC-based custom-made program, which generated histogram bars representing the cumulative number of spikes in consecutive $10 \mathrm{~s}$ bins. Noradrenergic neurons in the LC were identified by the following electrophysiological criteria: a spontaneous and regular discharge, a slow firing rate and a positive-negative biphasic waveform of 3-4 ms duration (Andrade \& Aghajanian 1984). We only recorded cells that showed stable firing rates between 0.4 and 1.5 $\mathrm{Hz}$ for at least 3-5 min and strong inhibitory effects induced by ME (0.8 mM, $1 \mathrm{~min}$ ) (higher than $80 \%$ ). Only one neuron was recorded per slice and only one slice was obtained from each animal. 


\subsection{Pharmacological procedures}

To characterize the effect of prostanoids in the LC neurons, we perfused increasing concentrations of the EP3/EP1 receptor agonist sulprostone (0.3-80 nM) and the endogenous derivatives of prostanoid system (PGE2 and misoprostol, $\quad 0.3 \quad \mathrm{nM}-1.28 \mu \mu \mathrm{M})$. Each concentration of the EP receptor agonists was perfused for at least $1 \mathrm{~min}$. We performed concentration/effect curves for the inhibitory effect of the agonists. In order to study the involvement of EP receptors in the effect observed with the EP receptor agonists, we used specific antagonist for the EP2 receptor (PF04418948), EP3 (L-798,106), and EP4 (L$161,982)$ at 3 and $10 \mu \mathrm{M}$. All the antagonists were perfused for at least 20-30 $\mathrm{min}$ before performing the concentration-effect curves for EP receptor agonists.

\subsection{Drugs and reagents}

Chloral hydrate was obtained from SigmaAldrich Química S.A. (Madrid, Spain). $\mathrm{Met}^{5}$ enkephalin (ME) was obtained from Bachem (Weil am Rhein, Germany). Sulprostone was purchased from Cayman Chemical (Michigan, USA). PF04418948, L-798,106 and L-161,982 were obtained from Tocris Bioscience (Bristol, UK). Drugs were dissolved in the final volume of aCSF just before each assay and applied by turning a threeway valve that switched from aCSF to the test solution. Stock solutions of the EP antagonist were made in DMSO stored at - 25 ${ }^{\circ} \mathrm{C}$ and, on the day of the experiment, diluted in aCSF to their final volume. The maximal final concentration of DMSO was lower than $0.1 \%$.

\subsection{Data analysis and statistics}

Values are expressed as the mean \pm standard error of the mean (S.E.M) of $\mathrm{n}$ experiments. The firing rate of LC cells was recorded before (baseline), during and after drug applications. The inhibitory effect of EP receptor agonists was calculated as follows:

$$
E(\%)=\frac{F R_{\text {pre }}-F R_{\text {post }}}{F R_{\text {basal }}} \cdot 100
$$

where $\mathrm{FR}_{\text {pre }}$ is the average firing rate for $60 \mathrm{~s}$ before application of each concentration, FR $\mathrm{F}_{\text {post }}$ is the average firing rate after the perfusion of each concentration, and FRbasal is the firing rate for 60 $\mathrm{s}$ of each cell at the beginning of the recording.

Changes induced by drugs in the firing rate were evaluated by a paired Student's $t$ test when compared within the same cell or by a twosample Student's t test when compared between different cells. Additionally, to evaluate the possible differences between groups the one-way ANOVA test was used. The level of significance was considered as $p=0.05$. Curve fitting analysis was performed by the computer program GraphPad Prism (version 5.0 for Windows, San Diego, CA, USA) to obtain the best simple nonlinear fit to the following three-parameter logistic equation:

$E=\operatorname{Emax} /\left[1+\left(E C_{50} / A\right)^{n}\right]$

where $E$ is the effect induced by each concentration of the EP3 agonist (A), Emax is the maximal effect, $\mathrm{EC}_{50}$ is the concentration of EP3 agonist needed to elicit a $50 \%$ of the maximal effect, and $n$ is the slope factor of the concentration-effect curve

\section{Conclusions}


In conclusion, LC neurons are regulated in an inhibitory manner by the prostanoid system likely through the EP3 receptor. Future experiments are required to characterize the relevance of other EP receptors to the modulation of the firing activity of LC cells.

\section{Acknowledgments}

This work was supported by the Ministerio de Ciencia e Innovación [Grant SAF2008-03612] and the University of the Basque Country (UPV/EHU) [Grant GIU11/27 and Grant GIU14/29]. Pineda's research group takes part in a network unit supported by the University of the Basque Country [UFI 11/35]. A. Nazabal was supported by a predoctoral fellowship from the Basque Government. The experiments comply with the current laws of Spain.

\section{Author Contributions}

A. Nazabal performed the research and analysed the data. A. Mendiguren and J. Pineda designed the research study. All mentioned authors wrote the manuscript.

\section{Conflicts of Interest}

The authors declare no conflict of interest.

\section{References and Notes}

Almeida, M.C. et al., 2004. Thermoeffector neuronal pathways in fever: a study in rats showing a new role of the locus coeruleus. The Journal of physiology, 558(Pt 1), pp.283-94.

Andrade, R. \& Aghajanian, G.K., 1984. Locus coeruleus activity in vitro: intrinsic regulation by a calcium-dependent potassium conductance but not alpha 2-adrenoceptors. The Journal of neuroscience, 4, pp.161-170.

Ek, M. et al., 2000. Distribution of the EP3 prostaglandin E2 receptor subtype in the rat brain: Relationship to sites of interleukin-1 - Induced cellular responsiveness. Journal of Comparative Neurology, 428(August), pp.5-19.

Exner, H.J. \& Schlicker, E., 1995. Prostanoid receptors of the EP3 subtype mediate the inhibitory effect of prostaglandin E2 on noradrenaline release in the mouse brain cortex. NaunynSchmiedeberg's archives of pharmacology, 351(1), pp.46-52.

Fujino, H. \& Regan, J.W., 2006. EP 4 Prostanoid Receptor Coupling to a Pertussis Toxin- Sensitive Inhibitory G Protein. Molecular Pharmacology, 69(1), pp.5-10.

Hétu, P.-O. \& Riendeau, D., 2005. Cyclo-oxygenase-2 contributes to constitutive prostanoid production in rat kidney and brain. The Biochemical journal, 391, pp.561-566. 
Hutchinson, A.J., Chou, C., Israel, D.D., Xu, W., Regan, J.W., 2009. Activation of EP2 prostanoid receptors in human glial cell lines stimulates the secretion of BDNF. Neurochem int, 54(7), pp.439-446.

Ikeda-Matsuo, Y. et al., 2011. Inhibition of prostaglandin E2 EP3 receptors improves stroke injury via anti-inflammatory and anti-apoptotic mechanisms. Journal of Neuroimmunology, 238(1-2), pp.34-43.

Ito, S., Okuda-Ashitaka, E. \& Minami, T., 2001. Central and peripheral roles of prostaglandins in pain and their interactions with novel neuropeptides nociceptin and nocistatin. Neuroscience research, 41, pp.299-332.

Ito, Y. et al., 2000. The prostaglandin E series modulates high-voltage-activated calcium channels probably through the EP3 receptor in rat paratracheal ganglia. Neuropharmacology, 39, pp.181190.

Ji, R. et al., 2010. EP1 Prostanoid Receptor Coupling to G i / o Up-Regulates the Expression of Hypoxia-Inducible Factor-1 $\backsim$ through Activation of a Phosphoinositide-3 Kinase Signaling Pathway. Molecular Pharmacology, 77(6), pp.1025-1036.

Levi, G., Minghetti, L. \& Aloisi, F., 1998. Regulation of prostanoid synthesis in microglial cells and effects of prostaglandin E 2 on microglial functions. Biochimie, pp.899-904.

Liu, J.-F. et al., 2010. Cyclooxygenase-2 enhances alpha2beta1 integrin expression and cell migration via EP1 dependent signaling pathway in human chondrosarcoma cells. Molecular cancer, 9, p.43.

Martin, F. et al., 2007. Constitutive cyclooxygenase-2 is involved in central nociceptive processes in humans. Anesthesiology, 106(5), pp.1013-8.

Mendiguren, A. \& Pineda, J., 2004. Cannabinoids enhance N-methyl-D-aspartate-induced excitation of locus coeruleus neurons by CB1 receptors in rat brain slices. Neuroscience letters, 363, pp.1-5.

Mendiguren, A. \& Pineda, J., 2007. CB1 cannabinoid receptors inhibit the glutamatergic component of $\mathrm{KCl}$-evoked excitation of locus coeruleus neurons in rat brain slices. Neuropharmacology, 52, pp.617-625.

Nakagawa, T. et al., 2000. Possible involvement of the locus coeruleus in inhibition by prostanoid EP(3) receptor-selective agonists of morphine withdrawal syndrome in rats. European journal of pharmacology, 390(3), pp.257-66.

Nakamura, K. et al., 2001. Prostaglandin EP3 receptor protein in serotonin and catecholamine cell groups: a double immunofluorescence study in the rat brain. Neuroscience, 103(3), pp.763-75. 
Negishi, M. et al., 1995. Selective coupling of prostaglandin E receptor EP3D to Gi and Gs through interaction of $\alpha$-carboxylic acid of agonist and arginine residue of seventh transmembrane domain. Journal of Biological Chemistry, 270, pp.16122-16127.

Sugimoto, Y. \& Narumiya, S., 2007. Prostaglandin E receptors. Journal of Biological Chemistry, 282(16), pp.11613-11617.

Yagami, T., Koma, H. \& Yamamoto, Y., 2015. Pathophysiological Roles of Cyclooxygenases and Prostaglandins in the Central Nervous System. Mol Neurobiol, DOI 10.100.

Yaksh, T.L. et al., 2001. The acute antihyperalgesic action of nonsteroidal, anti-inflammatory drugs and release of spinal prostaglandin E2 is mediated by the inhibition of constitutive spinal cyclooxygenase-2 (COX-2) but not COX-1. The Journal of neuroscience : the official journal of the Society for Neuroscience, 21(16), pp.5847-5853.

Zhang, J. \& Rivest, S., 1999. Distribution, regulation and colocalization of the genes encoding the EP 2 - and EP 4 -PGE 2 receptors in the rat brain and neuronal responses to systemic inflammation. European Journal of Neuroscience, 11, pp.2651-2668.

(C) 2015 by the authors; licensee MDPI, Basel, Switzerland. This article is an open access article distributed under the terms and conditions defined by MDPI AG, the publisher of the Sciforum.net platform. Sciforum papers authors the copyright to their scholarly works. Hence, by submitting a paper to this conference, you retain the copyright, but you grant MDPI AG the non-exclusive and unrevocable license right to publish this paper online on the Sciforum.net platform. This means you can easily submit your paper to any scientific journal at a later stage and transfer the copyright to its publisher (if required by that publisher). (http://sciforum.net/about ). 